\title{
Characterization of melting properties of several Fe-C model potentials
}

\author{
Mykhailo Melnykov ${ }^{1}$ and Ruslan L. Davidchack ${ }^{1}$ \\ ${ }^{1}$ Department of Mathematics, University of Leicester, Leicester, LE1 7RH, UK
}

\begin{abstract}
We use the coexisting phases approach to calculate melting phase diagrams of several Fe-C interaction potentials, such as Embedded Atom Method (EAM) potential of Lau et al. [Phys. Rev. Lett. 98, 215501 (2007)], EAM potential of Hepburn and Ackland [Phys. Rev. B 78, 165115 (2008)], and two flavours of the Analytic Bond Order potential (ABOP) of Henriksson and Nordlund [Phys. Rev. B 79, 144107 (2009)]. Melting of both bcc (ferrite) and fcc (austenite) crystals is investigated with $\mathrm{C}$ concentrations up to $5 \mathrm{wt} \%$. The results are compared with the experimental data and suggest that the potential of Hepburn and Ackland is the most accurate in reproducing the melting phase diagram of the ferrite, although the austenite cannot be stabilized at any $\mathrm{C}$ concentration for this potential. The potential of Lau et al. yields the best qualitative agreement with the real phase diagram in that the ferrite-liquid coexistence at low $\mathrm{C}$ concentrations is replaced by the austenite-liquid coexistence at higher $\mathrm{C}$ concentrations. However, the crossover $\mathrm{C}$ concentration is much larger and the ferrite melting temperature is much higher than in the real Fe-C alloy. The ABOP of Henriksson and Nordlund without the Ziegler-Biersack-Littmark (ZBL) correction correctly predicts the relative stability of ferrite and austenite at melting, but significantly underestimates the solubility of $\mathrm{C}$ in the solid phases, while the same potential with the ZBL correction predicts the austenite to be more stable compared to the ferrite at all $\mathrm{C}$ concentrations near the melting transition.
\end{abstract}

\section{Highlights}

- Coexisting phases approach is used to compute the phase diagrams of Fe-C model alloys;

- Melting phase diagrams are computed for the fcc and bcc crystals;

- Fe-C models described by Embedded Atom Method and Analytic Bond Order potentials;

- The results are compared with the melting phase diagram of the real Fe-C alloy.

Keywords: Molecular dynamics; coexisting phases simulation; melting phase diagram; Embedded Atom Model potential; Analytic Bond Order Potential; austenite; ferrite.

PACS numbers:

\section{INTRODUCTION}

Iron and iron-based alloys are of considerable interest for the materials modelling community because of the immense technological importance of steels. Understanding the melting properties of these materials is essential since they determine microstructure of steels which is important in applications such as casting and welding. Molecular simulation of such materials can offer insight into the formation and evolution of microstructures during solidification. However, most of the interatomic potentials developed for iron and iron-based alloys are optimized at lower temperatures, and the melting properties of these model potentials are rarely explored.

In this work we investigate the melting properties of several Fe-C model potentials. We study the Fe-C alloy with relatively low $\mathrm{C}$ concentrations, up to about 20 at $\%$ (5 wt\%), and therefore explore the melting of ferrite and austenite, but not of cementite. The experimental phase diagram of the real Fe-C system is shown in Figure 1. The key feature of the diagram at temperatures near melting is the interplay between the bcc phase, known as the $\delta$-iron or $\delta$-ferrite, at low $\mathrm{C}$ concentrations and the fcc phase, or $\gamma$-iron (austenite), at higher $\mathrm{C}$ concentrations ${ }^{1}$. For pure iron $(C=0)$, the bcc crystal ( $\delta$-ferrite) is stable from the melting temperature of $1811 \mathrm{~K}$ down to $1667 \mathrm{~K}$, where it is replaced by a more stable fcc crystal (austenite).

Carbon is an interstitial impurity in both fcc and bcc iron crystals, occupying predominantly octahedral sites (middle of edges and the centre of the fcc unit cell and

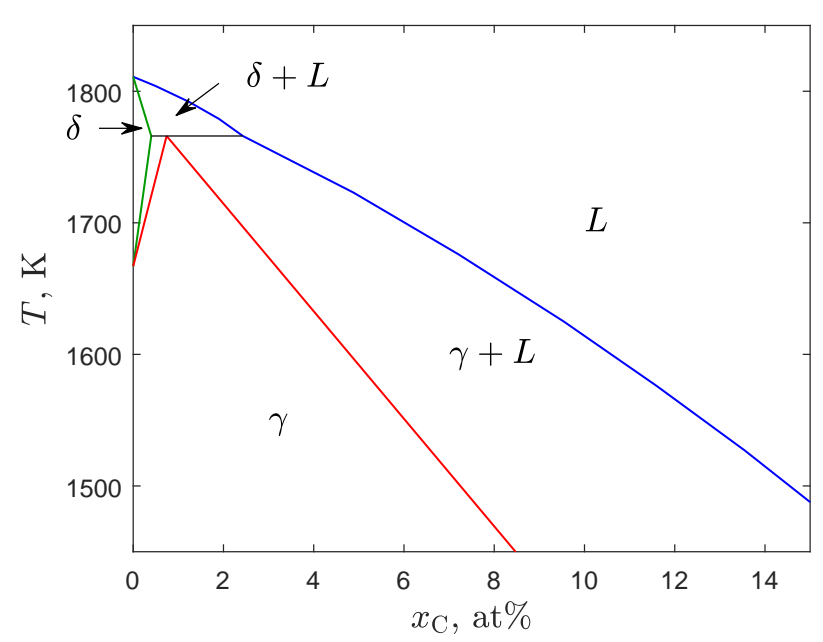

FIG. 1: Experimental temperature-concentration phase diagram of the Fe-C system. Adapted from Ref. 1

middle of faces and edges of the bcc unit cell). Since carbon more readily dissolves in the fcc crystal, its presence stabilises the $\gamma$ phase relative to the $\delta$ phase, which is also confirmed in the measurement of relative free energies of bcc and fcc Fe-C crystals in model systems ${ }^{2}$. This manifests itself in the disappearance of the $\delta$ phase from the phase diagram at $\mathrm{C} \gtrsim 0.4$ at $\%$ and the replacement of the $\delta$-liquid coexistence with the $\gamma$-liquid coexistence at $T<1766 \mathrm{~K}$ (below the horizontal black line in Figure 1). 
While we do not expect that a model Fe-C potential will reproduce exactly the phase diagram structure of the real system, it would be desirable to obtain at least a qualitative agreement with the experimental results. Unfortunately, to the best of our knowledge, most of the studies to date have focused on the properties of the low temperature $\alpha$-ferrite ${ }^{3-5}$, while those where the melting of $\mathrm{Fe}$ or $\mathrm{Fe}-\mathrm{C}$ systems was considered, only one phase melting was investigated ${ }^{6}$. Therefore, to the best of our knowledge, the present investigation is the first which investigates the interplay between the melting properties of fcc and bcc phases in the Fe-C system.

The rest of the paper is organised as follows. In Section II we present the methodology of calculating the solid-liquid coexistence properties of the $\mathrm{Fe}-\mathrm{C}$ system. In Section III we briefly discuss the model potentials which we investigate in this work. The results are presented in Section IV, with conclusions in Section V.

\section{METHODOLOGY}

Phase diagrams for multicomponent systems can be obtained by a combination of the common tangent construction $^{7}$ and the Gibbs-Duhem integration ${ }^{8}$. The common tangent construction requires calculation of Gibbs free energies of different phases as functions of temperature, pressure, and concentrations of various components $^{9}$. Such calculations are usually quite complicated and computationally demanding, especially for dense solid and liquid phases. Nevertheless, they have been used to determine solid-liquid and solid-solid phase boundaries in Fe-based metal alloys. For example, Lopasso et $a l^{10}$ used this approach to determine the Fe-Cu phase diagram, while Sak-Saracino and Urbassek calculated the fcc-bcc phase boundary in a model Fe-C alloy $^{2}$ with different $\mathrm{C}$ concentrations.

A simpler alternative to the evaluation of the Gibbs free energies is to carry out a simulation of the two phases coexisting within the same simulation box and separated by an interface. If the simulation conditions are close to coexistence, then the two phases will remain in relative equilibrium during the simulation. Otherwise, a net melting or freezing will be observed in the simulation, depending on whether the simulation conditions are within the liquid or solid phase, respectively. By performing simulations at a number of different conditions, it is possible to determine the location of the phase coexistence line. This approach was successfully applied to determine the solid-liquid coexistence conditions in hard and soft spheres and Lennard-Jones system ${ }^{11-13}$, several models of water ${ }^{14,15}$, many metals ${ }^{6,16,17}$, as well as more complex molecular crystals $^{18}$.

Typically, the method of coexisting phases cannot be used directly to investigate the melting properties of multicomponent materials due to very slow inter-diffusion of the components, especially in the solid phase. However, in the case of the Fe-C alloys with low $\mathrm{C}$ concentrations, the direct coexistence method benefits from the fact that carbon diffuses relatively well both in the liquid and solid phases at temperatures close to melting. Therefore, we have used the coexisting phases approach in our simula- tions.

To set up the coexistence simulations, we first prepared separate solid (fcc or bcc) and liquid systems with given $\mathrm{C}$ concentrations. Carbon atoms were initially placed randomly at the octahedral sites in the solid phase. The solid system was equilibrated at given pressure and temperature using Berendsen thermostat and anisotropic barostat, where the simulation box dimensions, $L_{x}, L_{y}$, and $L_{z}$ are allowed to fluctuate independently. The liquid system was initialised in the same way as the solid, but then melted in a simulation run with the thermostat at $3000 \mathrm{~K}$, followed by an equilibration run at the same temperature as the solid system, with anisotropic Berendsen barostat where only $L_{z}$ is allowed to vary. The typical size of the solid and liquid systems was about $L_{x} \approx L_{y} \approx 40 \AA$, $L_{z} \approx 80 \AA$ with about $10000 \mathrm{Fe}$ atoms. The orientation of bcc and fcc crystals in the solid system was (100). The two systems were then combined in a single simulation volume with $1 \AA$ gaps between the two systems along the $\mathrm{z}$ axis, preceded by a small constant volume scaling of the liquid system in order to match the $L_{x}$ and $L_{y}$ dimensions of the solid system. Potential energy minimisation was performed in order to remove high-energy states of any solid and liquid atoms that happen to be too close. Coexistence simulations were carried out on the combined solid-liquid system of approximate dimension $L_{x} \approx L_{y} \approx 40 \AA, L_{z} \approx 160 \AA$ with about $20000 \mathrm{Fe}$ atoms. The system size is relatively large in order to have a sufficient range of equilibration through freezing/melting at the solid-liquid interfaces and relatively stable locations of the interfaces in the equilibrated systems, which exhibit capillary fluctuations. In all the simulation runs the barostat pressure was 1 atm.

Coexistence conditions for the solid and liquid phases of the $\mathrm{Fe}-\mathrm{C}$ system are characterised by the equality of temperature, $T_{\mathrm{s}}=T_{1}$, pressure $P_{\mathrm{s}}=P_{1}$, and chemical potentials of the two components: $\mu_{\mathrm{Fe}, \mathrm{s}}=\mu_{\mathrm{Fe}, 1}$ and $\mu_{\mathrm{C}, \mathrm{s}}=\mu_{\mathrm{C}, \mathrm{l}}$. While temperature and pressure equilibrate to the given thermostat and barostat conditions relatively quickly through the balance of kinetic energies and forces, the equilibration of chemical potentials is achieved through much slower processes of melting/freezing at the solid-liquid interfaces and the diffusion of carbon atoms between the two phases across the interfaces. When the initial conditions are far from coexistence, we observe significant freezing or melting at the interfaces, up to a complete freezing or melting of the whole system. Otherwise, the system reaches equilibrium coexistence conditions after sufficiently long simulations with significant fractions of both phases present in the simulation volume. Typical simulation runs of 8-10 ns were required to reach the equilibrium conditions, but in some cases (especially at lower temperatures) the runs were extended to $20-30 \mathrm{~ns}$ in order to allow the $\mathrm{C}$ concentrations in the solid and liquid to equilibrate.

In order to ascertain that equilibrium $\mathrm{C}$ concentrations were reached in the solid and liquid phases, we also performed control simulations starting from initial C concentrations on the opposite side of the coexistence line. For example, if during equilibration we observed net flow of $\mathrm{C}$ atoms from solid to liquid due to initial excess of $\mathrm{C}$ atoms in the solid compared to the coexistence concen- 


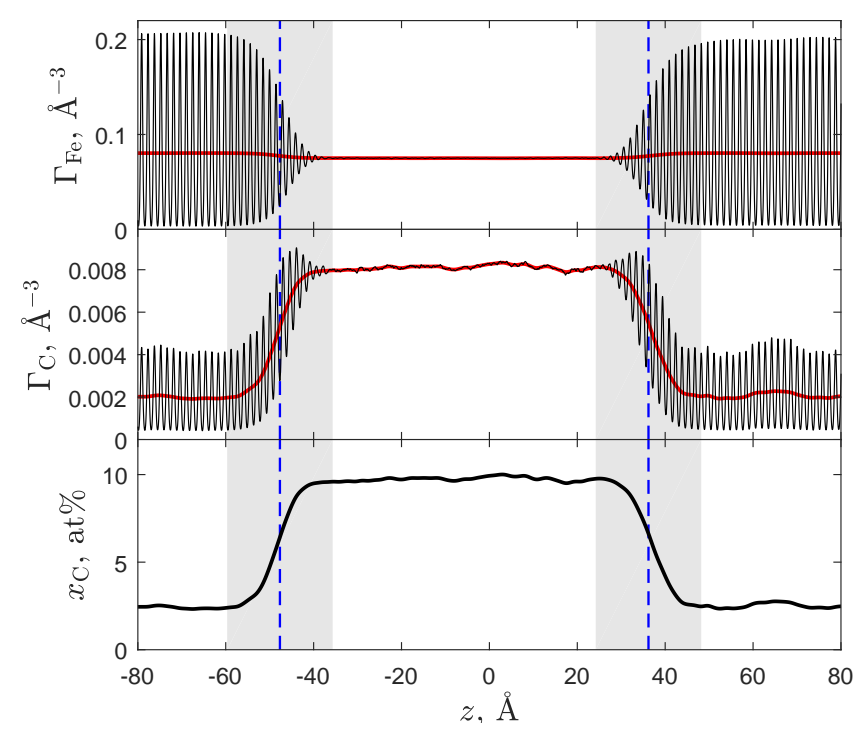

FIG. 2: Average number densities and $\mathrm{C}$ concentration profiles for the bcc-liquid system at $T \approx 2030 \mathrm{~K}$ for the $\mathrm{Fe}-\mathrm{C}$ model potential by Lau et al. ${ }^{3}$. Thick red lines indicate filtered density profiles, $\bar{\Gamma}(z)$; vertical dashed blue lines indicate positions of the solid-liquid interfaces; shaded areas indicate interfacial regions.

tration, we initiated another simulation run at the same temperature and lower $\mathrm{C}$ concentrations in the solid, observing the opposite net flux during equilibration and convergence to the same, within statistical uncertainty, $\mathrm{C}$ concentrations in the solid and liquid phases as in the previous run.

Once the equilibrium of the solid-liquid system was reached, the measurements of $\mathrm{Fe}$ and $\mathrm{C}$ number densities, $\Gamma_{\mathrm{Fe}}$ and $\Gamma_{\mathrm{C}}$, in the bulk solid and liquid phases at coexistence were taken during a subsequent $2 \mathrm{~ns} N V T$ simulation run with the Nosé-Hoover thermostat. Fe and $\mathrm{C}$ number density profiles as functions of $z$, averaged in the bins of width approx. 1/10-th of the interlayer spacing in the solid phase. An example of the time-averaged $\mathrm{Fe}$ and $\mathrm{C}$ density profiles in the bcc-liquid system is shown Figure 2. To suppress the density oscillations in the solid and determine the average solid and liquid densities, a finite impulse response filter was applied to the density profiles $^{11}$

$$
\bar{\Gamma}\left(z_{i}\right)=\sum_{j=-n}^{n} w_{j} \Gamma\left(z_{i+j}\right), \quad w_{i}=\frac{\mathrm{e}^{-5(i / n)^{2}}}{\sum_{j=-n}^{n} \mathrm{e}^{-5(j / n)^{2}}},
$$

where $z_{i}=z+i \Delta z$ with $\Delta z$ being the width of the bins. The factor 5 in the exponent and the parameter $n=20$ (twice the number of bins in the crystal interlayer spacing) have been determined to be optimal for filtering out the crystal density oscillations. The filtered profiles are shown in Figure 2 with the thick red lines. The $\mathrm{C}$ concentration profile in the bottom plot of the Figure was calculated from the filtered density profiles

$$
x_{\mathrm{C}}(z)=\frac{\bar{\Gamma}_{\mathrm{C}}(z)}{\bar{\Gamma}_{\mathrm{Fe}}(z)+\bar{\Gamma}_{\mathrm{C}}(z)} .
$$

The positions of the interfaces, indicated by the vertical dashed blue lines, were identified with the $z$ coordinate where the magnitude of the Fe density profile oscillations was approximately half of that in the bulk solid. The shaded areas indicate interfacial regions. The width of the regions, $24 \AA$, was chosen large enough to remove its effect on the computed average bulk properties. The average $\mathrm{Fe}$ and $\mathrm{C}$ number densities were obtained from the unshaded intervals of the density profiles.

We have conducted our simulations with the DL_POLY package $^{19}$, which we have modified to enable certain features of the interaction potentials used in this study and to monitor equilibration of the solid-liquid system.

\section{FE-C MODEL POTENTIALS}

Several different model potentials for the Fe-C system can be found in the literature ${ }^{2-5,20-22}$. Since most of these potentials have been developed to model ferrite or cementite at temperatures far below melting, their ability to describe the properties of Fe-C system at temperatures close to melting is not known. For our computation of the melting phase diagrams, we chose to investigate the Embedded Atom Model (EAM) potentials of Lau et al. ${ }^{3}$ and of Hepburn and Ackland ${ }^{21}$, and the Analytic Bond Order (ABOP) potential of Henriksson and Nordlund ${ }^{22}$.

Among the $\mathrm{Fe}-\mathrm{C}$ potentials that we did not investigate due to the limitations of our DL_POLY implementation of the interaction models we would like to mention the 2nd-Nearest Neighbour Modified Embedded Atom Model (MEAM) by Lee ${ }^{5}$ and the model proposed by SakSaracino and Urbassek ${ }^{2}$, which combines the Fe-Fe EAM parametrisation by Meyer and $\mathrm{Entel}^{23}$, pairwise potential of Johnson et al. ${ }^{24}$ or Rosato ${ }^{25}$ for the Fe-C interactions, and the Tersoff potential for the $\mathrm{C}-\mathrm{C}$ interactions ${ }^{26}$. Even though the melting phase diagrams for these models have not been calculated, they may be suitable for modelling melting properties of the bcc and fcc Fe-C alloy at low $\mathrm{C}$ concentrations. The relevant studies for the MEAM potential are by Timmerscheidt et $a .^{27}$ on carbon diffusion in fcc iron, and characterisation of cementite and cementite-liquid inteface by Liyanage et al. ${ }^{28}$, while the model proposed by Sak-Saracino and Urbassek has been used to study the $\alpha \leftrightarrow \gamma$ solid-solid transitions in bulk ${ }^{29}$ and in nanowires ${ }^{30}$.

\section{A. EAM potential by Lau et al. ${ }^{3}$}

This EAM potential for the Fe-C system was developed primarily for the accurate description of the bcc $\alpha$-ferrite supersaturated in $\mathrm{C}$ with particular emphasis on accurate prediction of configurations and energetics of point defect clusters. The form of the EAM potential and the Fe-Fe interaction parameters were those proposed by Rosato ${ }^{25}$, while the $\mathrm{Fe}-\mathrm{C}$ and $\mathrm{C}-\mathrm{C}$ interactions were parametrised to match the experimental and DFT results for the configurations and formation energies of different carbonvacancy point defect clusters. As far as we know, this potential has not been tested at higher temperatures or for modelling the austenite phase, but the simple EAM form of the potential, which is easily implementable in 
DL_POLY, makes it an attractive candidate for our investigation.

\section{B. EAM potential by Hepburn and Ackland ${ }^{21}$}

This potential was also developed for modelling the $\alpha$ ferrite phase of the $\mathrm{Fe}-\mathrm{C}$ system with correct description of the interaction of iron and carbon across a wide range of defect environments, particularly reflecting the large solvation energy of carbon in iron. The Fe-Fe interaction parameters were inherited from Ref. 31, which is based on the EAM potential by Mendelev et al. ${ }^{32}$, developed with the view of modelling both the bcc crystal and liquid phases of iron. As such, it is expected to be well suited for modelling the bcc-liquid coexistence conditions.

\section{ABOP by Henriksson and Nordlund ${ }^{22}$}

The Analytic Bond-Order potential (ABOP) formalism has a functional form similar to that of Brenner ${ }^{33}$ and Tersoff ${ }^{34}$ potentials, and has been used to describe interaction of elements with different bonding types, including metals, semiconductors, and their combinations. Henriksson and Nordlund ${ }^{22}$ adopted the Fe-Fe ABOP parameters from Müller, Erhart, and Albe ${ }^{35}$, the C-C interaction parameters from Brenner ${ }^{33}$, and derived the $\mathrm{Fe}-\mathrm{C}$ parameters to match relatively well the properties of cementite $\mathrm{Fe}_{3} \mathrm{C}$, carbides $\mathrm{Fe}_{7} \mathrm{C}_{3}$ and $\mathrm{Fe}_{4} \mathrm{C}$, and some point defect systems available from experiments and density functional theory calculations.

The $\mathrm{Fe}-\mathrm{Fe} \mathrm{ABOP}^{35}$ is, to the best of our knowledge, the only Fe potential designed to reproduce the $\alpha-\gamma-\delta$ solid phase transitions in pure iron. According to Ref. 35, with increasing temperature, the model predicts a bccfcc transition at $1030 \mathrm{~K}$ (compared to $1184 \mathrm{~K}$ for the $\alpha-\gamma$ transition of the real $\mathrm{Fe}$ ) and an fcc-bcc transition at $2210 \mathrm{~K}$ followed by the bcc melting point at $2270(20) \mathrm{K}$, corresponding to $1665 \mathrm{~K}$ for the real $\gamma-\delta$ transition followed by the $1811 \mathrm{~K}$ melting temperature of real $\delta$-iron.

Our solid-liquid coexistence simulations yield the bcc phase melting temperature of $2284(5) \mathrm{K}$. We have also determined the fcc melting temperature in the fcc-liquid coexistence simulations, which is $2270(5) \mathrm{K}$. The fact that the melting temperature of the fcc phase is lower than that of the bcc phase indicates that the fcc phase is less stable at these temperatures. (Note that, even though the fcc phase is less stable than bcc, the fcc phase in the fcc-liquid coexistence simulations is stabilised by the periodic boundary conditions commensurate with the fcc crystal.)

In order to simulate high-energy events that occur, for example, during sputtering, Henriksson and Nordlund introduced a short-distance modification of the ABOP potential

$$
\tilde{V}(r)=F(r) V_{\mathrm{ABOP}}(r)+[1-F(r)] V_{\mathrm{ZBL}}(r),
$$

where $r$ is the distance between a pair of atoms, $V_{\mathrm{ABOP}}(r)$ is the original $\mathrm{ABOP}$ potential, $V_{\mathrm{ZBL}}(r)$ is the Ziegler-Biersack-Littmark potential ${ }^{36}$, and $F(r)=$ $\left(1+\exp \left[-b_{f}\left(r-r_{f}\right)\right]\right)^{-1}$ is the Fermi-Dirac function with two fitting parameters $r_{f}$ and $b_{f}$.

Henriksson and Nordlund state ${ }^{22}$ that the function $F(r)$ is sufficiently short-ranged, so that the equilibrium properties of the original ABOP potential are maintained. However, in our preliminary investigations we have noticed that the ZBL modification has significant effect on the melting properties of the model. In particular, the melting temperature of the bcc phase increases by about $60 \mathrm{~K}$ to become $2341(5) \mathrm{K}$, while that of the fcc phase increases by about $100 \mathrm{~K}$ to become 2371 (5) K. As a result, the fcc phase becomes more stable than bcc and so the modified ABOP model no longer predicts the fcc-bcc solid phase transition, which corresponds to the $\gamma-\delta$ transition in real iron. This, of course, has fundamental consequences for the qualitative structure of the $\mathrm{Fe}-\mathrm{C}$ phase diagram.

The reason that the ZBL modification has an effect on the equilibrium properties of the ABOP model of iron is that at temperatures around $2300 \mathrm{~K}$ the iron atoms approach each other to within about $1.9 \AA$. With parameters $r_{f}=0.9 \AA$ and $b_{f}=2.9 \AA^{-1}$ for the Fe-Fe interactions, the value $1-F(1.9) \approx 0.06$ is still relatively large, so that the ZBL correction, which is much more repulsive than the ABOP at these distances, has significant influence on the interaction of iron atoms at short distances. At the same time, parameters of the switching function for the $\mathrm{Fe}-\mathrm{C}$ and $\mathrm{C}-\mathrm{C}$ interactions are such that the ZBL correction is insignificant at the minimal approach distances between $\mathrm{Fe}$ and $\mathrm{C}$ atoms.

In order to provide a link with the qualitatively correct description of pure Fe phase diagram by the ABOP potential of Müller, Erhart, and $\mathrm{Albe}^{35}$, in this paper, we present $\mathrm{Fe}-\mathrm{C}$ melting phase diagram for the ABOP model both with and without the ZBL correction.

\section{RESULTS}

Here we present results of our calculations of the fcc and bcc Fe-C alloy melting phase diagrams for the model potentials discussed in the previous section. We present the phase diagram in the temperature versus $\mathrm{C}$ concentration plane, as well as the solid and liquid densities of Fe at coexistence conditions.

\section{A. Lau et al. potential}

Figure 3 shows results for the melting phase diagram obtained using Lau et al. EAM potential ${ }^{3}$. The corresponding densities of solid and liquid phases at the coexistence conditions as functions of temperature are shown in Figure 4. The melting phase diagram for this model potential agrees qualitatively with the experimental phase diagram in that the bcc-liquid coexistence at lower $\mathrm{C}$ concentrations is replaced by the fcc-liquid coexistence at higher $\mathrm{C}$ concentrations. However, quantitative agreement with the experiment is rather poor. First of all, the melting temperature of pure Fe bcc solid is about $2400 \mathrm{~K}$, which is about $600 \mathrm{~K}$ higher than experimental melting temperature of pure Fe, so the whole 


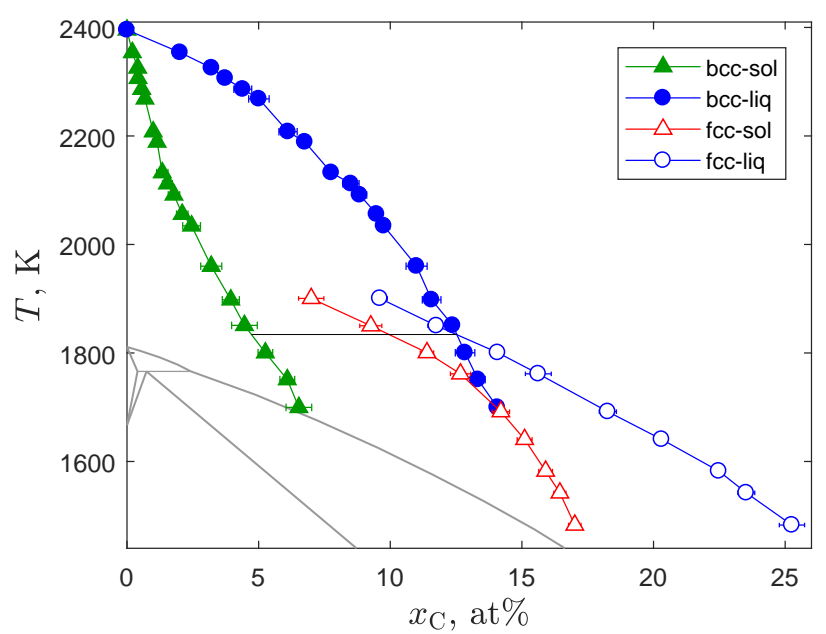

FIG. 3: Phase diagram for the EAM potential by Lau et $a .^{3}$ The horizontal black line indicates the estimated crossover between the bcc-liquid and fcc-liquid coexistence conditions. Horizontal error bars indicate $95 \%$ confidence intervals in the measured $\mathrm{C}$ concentrations. Confidence intervals in the temperature measurements are smaller than the size of the symbols. The data points are connected as a guide to the eye only. The thin black lines indicate the experimental phase diagram.

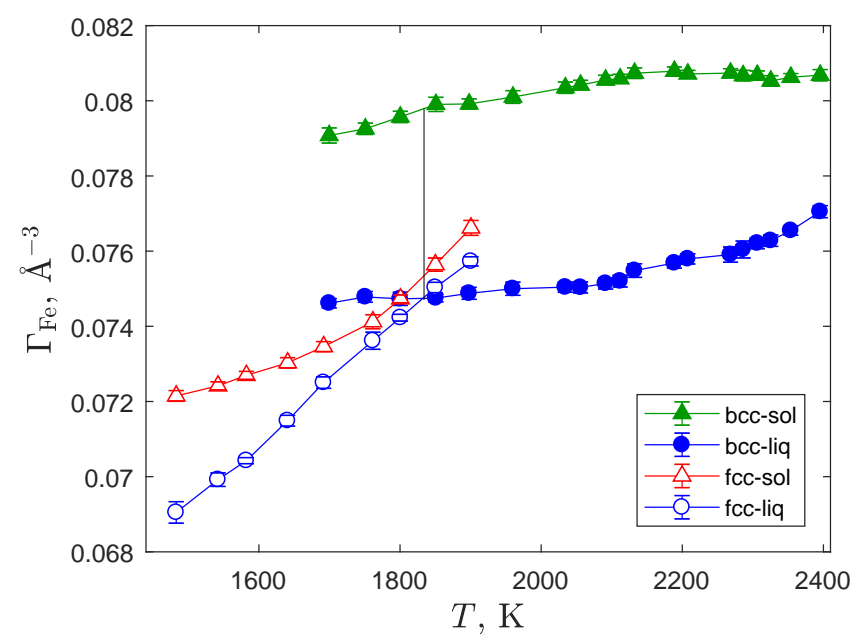

FIG. 4: Fe number density along the solid-liquid coexistence lines for the EAM potential by Lau et al. ${ }^{3}$ The vertical black line indicates the estimated crossover between the bcc-liquid and fcc-liquid coexistence conditions. Vertical error bars indicate $95 \%$ confidence intervals in the measured number densities of Fe. The data points are connected as a guide to the eye only.

bcc phase diagram is shifted to higher temperatures compared to experimental data. This reflects the properties of Rosato's parametrisation ${ }^{25}$ of the EAM potential for pure Fe.

Also, the bcc-liquid coexistence phase extends to much higher $\mathrm{C}$ concentrations than the $\delta$-iron-liquid coexistence in the real system. The cross-over from the bccliquid to the fcc-liquid coexistence conditions appear as

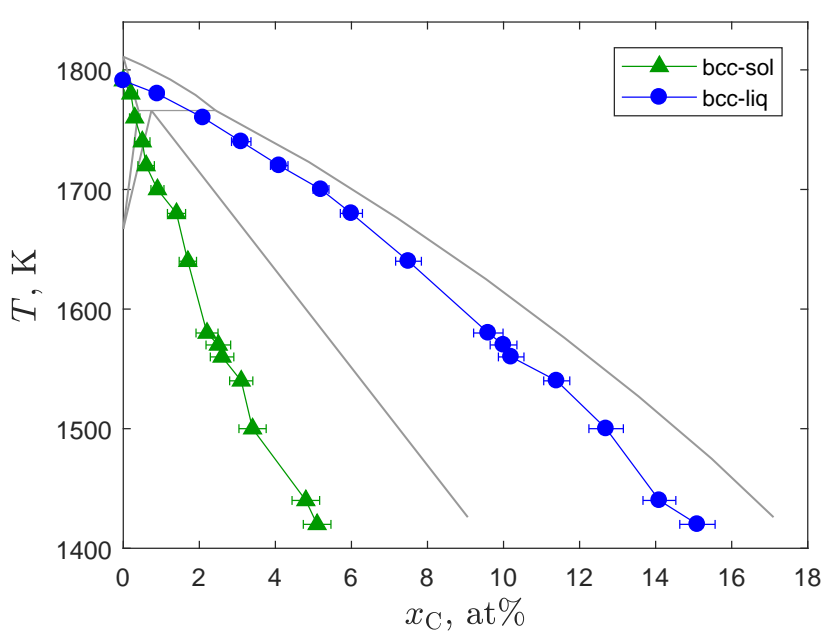

FIG. 5: Phase diagram of the EAM potential by Hepburn and Ackland ${ }^{21}$. Other notations are explained in Figure 3 caption.

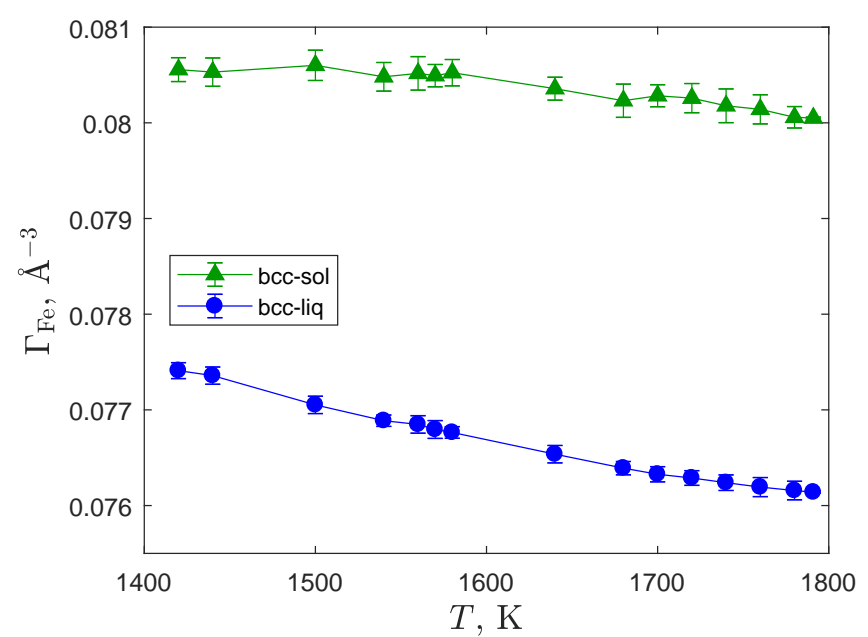

FIG. 6: Fe number density along the solid-liquid coexistence lines for the EAM potential by Hepburn and Ackland ${ }^{21}$. Other notations are explained in Figure 4 caption.

the intersection of the bcc-liq and fcc-liq coexistence lines in Figure 3, as well as the intersection of the liquid phase density lines as functions of temperature in Figure 4.

\section{B. Hepburn and Ackland potential}

The melting phase diagram for the EAM potental of Hepburn and Ackland ${ }^{21}$ is shown in Figure 5, with the corresponding densities of the phases at coexistence shown in Figure 6. It can be seen that this potential is much better than that of Lau et al. at reproducing the melting properties of the bcc phase. However, the fcc phase is not shown because, according to our simulations, the fcc crystal is structurally unstable at all the studied temperatures and $\mathrm{C}$ concentrations. 


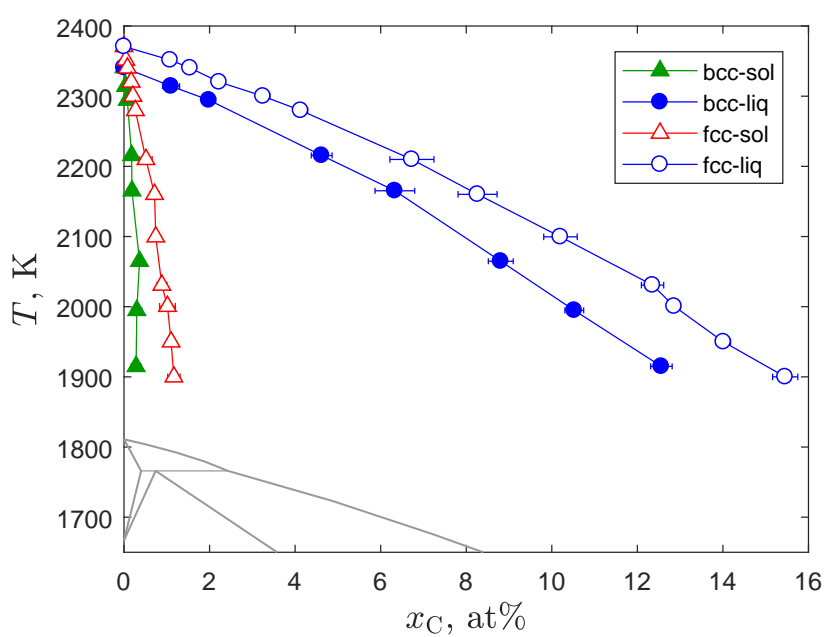

FIG. 7: Phase diagram of the ABOP potential by Henriksson and Nordlund ${ }^{22}$. Other notations are explained in Figure 3 caption.

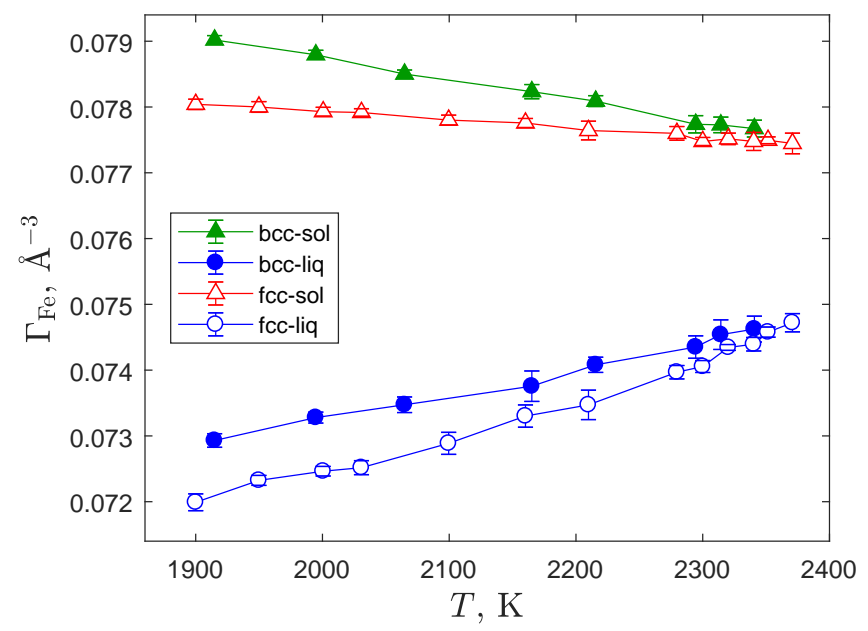

FIG. 8: Fe number density along the solid-liquid coexistence lines for the ABOP potential by Henriksson and Nordlund ${ }^{22}$. Other notations are explained in Figure 4 caption.

\section{ABOP by Henriksson and Nordlund}

Figure 7 reports results obtained with the Analytic Bond Order Potential proposed by Henriksson and Nordlund $^{22}$. The corresponding Fe densities at coexistence are shown in Figure 8. As discussed in Section III C, even though the Fe-Fe part of this potential is adopted from Müller, Erhart, and Albe ${ }^{35}$, which predicts the fcc-bcc transition on increasing temperature near melting, the ZBL correction introduced by Henriksson and Nordlund modifies the short-range interaction between Fe atom, leading to a relative stabilisation of the fcc phase. As a result, the fcc phase of the Fe-C alloy is more stable than the bcc phase near melting at all studied $\mathrm{C}$ concentrations. Note that it is possible to reliably determine the melting properties of the bcc phase in the bcc-liquid coexistence simulations due to the stabilising effect of the

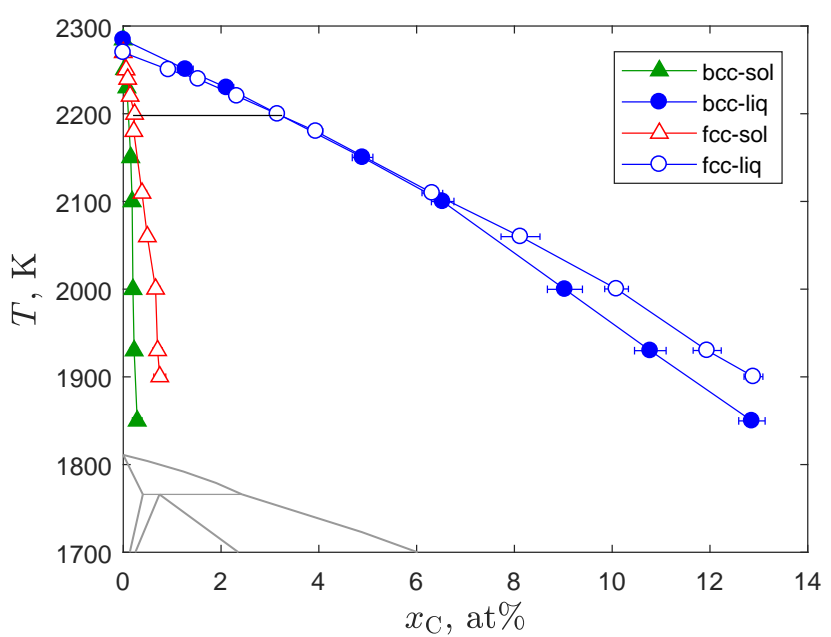

FIG. 9: Phase diagram of the ABOP potential by Henriksson and Nordlund ${ }^{22}$ without the ZBL correction. Other notations are explained in Figure 3 caption.

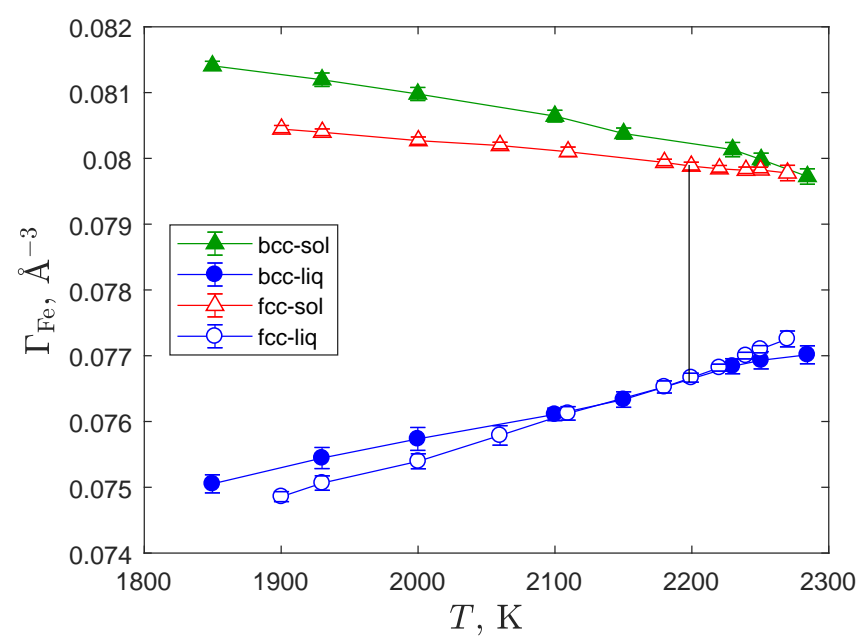

FIG. 10: Fe number density along the solid-liquid coexistence lines for the ABOP potential by Henriksson and Nordlund ${ }^{22}$ without the ZBL correction. Other notations are explained in Figure 4 caption.

periodic boundary conditions. So, unlike the potential of Hepburn and Ackland, where the fcc phase could not be stabilised at all, here the bcc phase is metastable with a sufficiently deep local free energy minimum to allow such stabilisation.

Compared to the experimental phase diagram, we see that, like in the case of Lau et al. potential, the melting of the Henriksson and Nordlund ABOP model is shifted to higher temperatures (due to the higher melting temperature of pure Fe in the model of Müller, Erhart, and Albe $\left.^{35}\right)$. Also, we see in Figure 7 that the solubility of $\mathrm{C}$ in the fcc and (metastable) bcc crystals at melting is much lower than in experiments. 


\section{ABOP by Henriksson and Nordlund without ZBL}

The melting phase diagram of the Henriksson and Nordlund ABOP without the ZBL correction is shown in Figure 9 with the corresponding Fe densities in Figure 10. We now observe a crossover from the bcc-liquid coexistence at higher temperatures to the fcc-liquid coexistence at lower temperatures. This is in qualitative agreement with the experimental phase diagram, although, as in the case with the ZBL, the melting temperature of both the bcc and fcc crystal alloys is too high and the $\mathrm{C}$ concentrations in the bcc and fcc crystals is too low, especially in the case of the fcc phase.

\section{CONCLUSIONS}

In this study we have demonstrated that the coexisting phases approach can be successfully used to calculate the melting phase diagram of model Fe-C alloys. The success of this approach is enabled by a sufficiently high diffusivity of carbon interstitials in the bcc and fcc crystal lattices of $\mathrm{Fe}$ atoms, which enables equilibration of $\mathrm{C}$ concentrations in 10-30 ns simulations runs.

Compared to the methods for determining phase diagrams through calculation of free energies ${ }^{9}$, the coexisting phases approach is much simpler to implement, even if it does require significant computational resources to carry out long simulations of relatively large systems equilibrated at many thermodynamic states. There exist various techniques that would allow using smaller system sizes and/or enable accelerated system equilibration and sampling of the equilibrium two-phase states. For example, one could utilise Grand or Semi-Grand Canonical ensemble Monte Carlo simulations to arrive at the coexistence conditions for the solid and liquid phases. One could also attempt $\mathrm{C}$ atom insertion/deletion moves to accelerate the $\mathrm{C}$ concentration equilibration or utilise Hamiltonian Replica Exchange approach ${ }^{37}$, where replicas with weaker Fe-C interactions would promote faster diffusion of $\mathrm{C}$ atoms throughout the system. These techniques, however, would be more complex to implement, and so we opt to use simpler direct methods, profiting from faster and larger modern computing facilities. Of course, for other solid-liquid systems, or for the $\mathrm{Fe}-\mathrm{C}$ system at higher pressures, were the equilibrium concentrations of multi-component systems cannot be reach through natural diffusion of the components, the use of such more complicated simulation techniques is unavoidable.

Using the direct coexisting phases approach, we have investigated three model potentials for the $\mathrm{Fe}-\mathrm{C}$ alloy with a specific focus on the ability of these models to reproduce the experimentally observed crossover from the $\delta$-liquid coexistence at low $\mathrm{C}$ concentrations to the $\gamma$ liquid coexistence at higher $\mathrm{C}$ concentrations. We observed that none of the three investigated model potentials reproduce all the features of the experimental melting phase diagram. Lau et al. EAM potential ${ }^{3}$ has correct qualitative features, but the fcc phase is much less stable and is stabilised at much higher $\mathrm{C}$ concentrations compared to the real alloy. Hepburn and Ackland EAM potential $^{21}$ is the best among the three at reproducing the bcc-liquid phase diagram, but fails to predict the existence of the stable fcc solid phase at higher $\mathrm{C}$ concentrations. Henriksson and Nordlund $\mathrm{ABOP}^{22}$ fails to predict the bcc-fcc crossover due to the ZBL correction, which they introduced to simulate high-energy events. Without this correction, the ABOP potential is qualitatively consistent with the real phase diagram, although it predicts higher than experimental melting temperatures of the bcc and fcc crystals as well as much lower solubility of $\mathrm{C}$ in the solid phase, especially the fcc crystal.

It is clear from our research that more work is required in order to develop a Fe-C alloy model potential that correctly predicts the melting properties of this system. Recently, a re-parametrisation of the MEAM potential has been proposed to improve the high-temperature properties of pure Fe, including the melting transition ${ }^{38}$. However, this work only focused on the bcc phase, while it is not clear whether the MEAM potential would be better than the investigated EAM and ABOP at predicting the crossover between the melting of the bcc and fcc phases. Such crossover may be better modelled by the Fe-C potential based on the Meyer and Entel EAM parameterisation for pure $\mathrm{Fe}^{23}$, as proposed by Sak-Saracino and Urbassek $^{2}$, since, similar to the ABOP model of pure Fe by Müller, Ergart, and Albe ${ }^{35}$, the Meyer-Entel model at low temperatures exhibits a metastable fcc phase, which is stabilised relative to the bcc phase at higher temperature $^{39}$ and/or by the presence of carbon ${ }^{2}$.

\section{ACKNOWLEDGMENTS}

This work was supported by the European Community's Seventh Framework Programme under grant agreement No: 229108. The computations were performed on the ALICE High Performance Computing Facilities at the University of Leicester. RLD did part of the work during a study leave granted by the University of Leicester.
${ }^{1} \mathrm{~W}$. Callister and D. Rethwisch, Materials Science and Engineering: An Introduction (Wiley, 2013), 9th ed., ISBN 9781118476543, URL https://books.google.co. uk/books?id=TmxbAgAAQBAJ.

2 E. Sak-Saracino and H. M. Urbassek, Philos. Mag. 94, 933 (2014), http://dx.doi.org/10.1080/14786435.2013.870359, URL http://dx.doi.org/10.1080/14786435.2013.
870359.

3 T. T. Lau, C. J. Först, X. Lin, J. D. Gale, S. Yip, and K. J. V. Vliet, Phys. Rev. Lett. 98, 215501 (2007).

4 M. Ruda, D. Farkas, and G. Garcia, Comput. Mater. Sci. 45, 550 (2009), ISSN 0927-0256, URL http://www. sciencedirect. com/science/article/B6TWM-4VDSCVV-1/2/ 
6ef04e7e33ab49c8228f 4c00cbfcd15f.

5 B.-J. Lee, Acta Materialia 54, 701 (2006).

${ }^{6}$ D. Y. Sun, M. Asta, and J. J. Hoyt, Phys. Rev. B 69, 174103 (2004)

7 A. Putnis, Introduction to Mineral Sciences (Cambridge University Press, 1992).

8 D. Kofke, J. Chem. Phys. 98, 4149 (1993).

${ }^{9}$ D. Frenkel and B. Smit, Understanding Molecular Simulation (Academic Press, New York, 2002), 2nd ed.

10 E. M. Lopasso, M. Caro, A. Caro, and P. E. A. Turchi, Phys. Rev. B 68, 214205 (2003).

11 R. L. Davidchack and B. B. Laird, J. Chem. Phys. 108, 9452 (1998).

12 J. R. Morris and X. Song, J. Chem. Phys. 116, 9352 (2002).

13 R. L. Davidchack and B. B. Laird, Phys. Rev. Lett. 94, $086102(2005)$

14 R. G. Fernández, J. L. F. Abascal, and C. Vega, J. Chem. Phys. 124, 144506 (2006).

15 R. L. Davidchack, R. Handel, J. Anwar, and A. V. Brukhno, J. Chem. Theory Comput. 8, 2383 (2012).

16 J. Morris, C. Wang, K. Ho, and C. Chan, Phys. Rev. B 49, 3109 (1994).

17 A. Potter and J. Hoyt, J. Cryst. Growth 327, 227 (2011), ISSN 0022-0248, URL http://www.sciencedirect.com/ science/article/pii/S0022024811004854.

18 J. Gerges and F. Affouard, The Journal of Physical Chemistry B 119, 10768 (2015), pMID: 26226388, http://dx.doi.org/10.1021/acs.jpcb.5b05557, URL http: //dx.doi.org/10.1021/acs.jpcb.5b05557.

19 W. Smith and I. T. Todorov, Mol. Simul. 32, 935 (2006).

20 C. Becquart, J. Raulot, G. Bencteux, C. Domain, M. Perez, S. Garruchet, and H. Nguyen, Computational Materials Science 40, 119 (2007), ISSN 0927-0256, URL http://www.sciencedirect. com/science/article/B6TWM-4MWGYHJ-2/2/ 52441d959a206c52e9a53bcecddec7b6.

21 D. J. Hepburn and G. J. Ackland, Phys. Rev. B 78, 165115 (2008).

${ }^{22}$ K. O. E. Henriksson and K. Nordlund, Phys. Rev. B 79 , 144107 (2009).

23 R. Meyer and P. Entel, Phys. Rev. B 57, 5140 (1998), URL https://link.aps.org/doi/10.1103/PhysRevB.57.5140.

${ }^{24}$ R. Johnson, G. Dienes, and A. Damask, Acta Metall. 12, 1215 (1964), ISSN 0001-6160, URL http://www.sciencedirect.com/science/article/ pii/0001616064901051.
25 V. Rosato, Acta Metallurg. 37, 2759 (1989).

26 J. Tersoff, Phys. Rev. B 39, 5566 (1989), URL https: //link.aps.org/doi/10.1103/PhysRevB.39.5566.

27 T. A. Timmerscheidt, J. von Appen, and R. Dronskowski, Comput. Mater. Sci. 91, 235 (2014), ISSN 09270256, URL http://www.sciencedirect.com/science/ article/pii/S0927025614002961.

28 L. S. I. Liyanage, S.-G. Kim, J. Houze, S. Kim, M. A. Tschopp, M. I. Baskes, and M. F. Horstemeyer, Phys. Rev. B 89, 094102 (2014), URL https://link.aps.org/doi/ 10.1103/PhysRevB.89.094102.

29 B. Wang, E. Sak-Saracino, N. Gunkelmann, and H. M. Urbassek, Comput. Mater. Sci. 82, 399 (2014), ISSN 09270256, URL http://www.sciencedirect.com/science/ article/pii/S0927025613006162.

30 B. Wang, E. Sak-Saracino, L. Sandoval, and H. M. Urbassek, Model. Simul. Mater. Sci. Eng. 22, 045003 (2014), URL http://stacks.iop.org/0965-0393/22/i= $4 / a=045003$.

31 G. Ackland, M. Mendelev, D. Srolovitz, S. Han, and A. Barashev, J. Phys.: Condens. Matter 16, S2629 (2004).

32 M. I. Mendelev, S. Han, D. J. Srolovitz, G. J. Ackland, D. Y. Sun, and M. Asta, Philosophical Magazine $\quad \mathbf{8 3}, 3977 \quad$ (2003), http://dx.doi.org/10.1080/14786430310001613264, URL http://dx.doi.org/10.1080/14786430310001613264.

33 D. W. Brenner, Phys. Rev. B 42, 9458 (1990), URL https : //link.aps.org/doi/10.1103/PhysRevB.42.9458.

34 J. Tersoff, Phys. Rev. Lett. 56, 632 (1986), URL https: //link.aps.org/doi/10.1103/PhysRevLett.56.632.

35 M. Müller, P. Erhart, and K. Albe, J. Phys.: Condens. Matter 19, 326220 (2007).

36 J. F. Ziegler, J. P. Biersack, and U. Littmark, The Stopping and Range of Ions in Solids (Pergamon, New York, 1985).

37 J. Hritz and C. Oostenbrink, J. Chem. Phys. 128, 144121 (2008), https://doi.org/10.1063/1.2888998, URL https: //doi.org/10.1063/1.2888998.

38 S. A. Etesami and E. Asadi, J. Phys. Chem. Solids 112, 61 (2018), ISSN 0022-3697, URL http://www. sciencedirect.com/science/article/ pii/S0022369717312039.

39 C. Engin, L. Sandoval, and H. M. Urbassek, Model. Simul. Mater. Sci. Eng. 16, 035005 (2008), URL http://stacks . iop.org/0965-0393/16/i=3/a=035005. 